\title{
Continued administration of antithrombotic agents during transperineal prostate biopsy
}

\author{
Toko Asano ${ }^{1}$, Shuichiro Kobayashi², Masataka Yano², Yukihiro Otsuka', Satoshi Kitahara² \\ ${ }^{1}$ Service of Urology, Omori Red Cross Hospital, Tokyo, Japan; ${ }^{2}$ Service of Urology, Tama-Nambu Chiiki \\ Hospital, Tokyo, Japan
}

\section{ABSTRACT}

Purpose: To determine the safety of continued administration of antithrombotic agents during transperineal (TP) prostate biopsy.

Patients and Methods: A total of 811 men who underwent transrectal ultrasound (TRUS)-guided TP biopsy from January 2008 to June 2012 at our two institutions were retrospectively analyzed. Among these 811 men, 672 received no antithrombotic agents (group I), 103 received and continued administration of antithrombotic agents (group II), and 36 interrupted administration of antithrombotic agents (group III). Overall complications were graded and hemorrhagic complications were compared (group I with group II) using propensity score matching (PSM) analysis.

Results: An overall complication rate of $4.6 \%$ was recorded. Hemorrhagic complications occurred in 1.8\% and they were virtually identical in all the three groups, and no severe hemorrhagic complications occurred. One patient in group III required intensive care unit admission for cerebral infarction. PSM analysis revealed no statistical difference between groups I and II with regard to the incidence of gross hematuria, perineal hematoma, and rectal bleeding. Multiple regression analysis revealed that hemorrhagic complications were associated with lower body mass index $\left(<21 \mathrm{~kg} / \mathrm{m}^{2}, \mathrm{P}=0.0058\right)$, but not with administration of antithrombotic agents.

Conclusions: Continued administration of antithrombotic agents does not increase the risk of hemorrhagic complications; these agents are well tolerated during TP biopsy.

\section{ARTICLE INFO}

\section{Key words:}

Prostate; Biopsy; Prostatic

Neoplasms; Anticoagulants

Int Braz J Urol. 2015; 41: 116-23

Submitted for publication:

December 16, 2013

Accepted after revision:

June 26, 2014

\section{INTRODUCTION}

Prostate cancer is the second most frequently diagnosed cancer and sixth leading cause of cancer-related death in men worldwide (1). Its incidence has increased substantially in recent years in many countries.

As a result of prolonged life span, the generation with a risk for prostate cancer coincides with that with a history of cardiovascular disease. Anticoagulant and/or antiplatelet agents (henceforth referred to as antithrombotic agents) are used for the prevention and treatment of thrombosis in patients with cardiovascular disease. Prostate cancer can be detected using a combination of digital rectal examination (DRE), serum prostate-specific antigen (PSA) concentration and magnetic resonance imaging (MRI). However, definitive diagnosis is only possible through a prostate needle biopsy. Therefore, antithrombotic agents are routinely discontinued before the procedure to avoid hemorrhagic complications $(2,3)$.

However, evidence has shown that discontinuation of antithrombotic agents can trigger serious 
and potentially fatal embolic complications. The odds ratio for ischemic stroke after aspirin interruption was 3.4 in patients with a history of ischemic heart disease (4). Of 526 patients in whom warfarin treatment was interrupted before dental surgery, five suffered serious embolic complications, and four of these patients died (5). The new edition of the American College Chest Physicians' authoritative clinical practice guidelines for the prevention and treatment of venous thromboembolism suggested continuation of warfarin for minor dermatological procedures and cataract surgery. They also suggested that thromboprophylaxis with aspirin in patients with cardiovascular disease should not be interrupted prior to major or minor surgery (6).

To avoid thrombosis triggered by discontinuation of antithrombotic agents, transrectal (TR) prostate biopsy under continued antithrombotic agents has been attempted with minor increase of biopsy-related bleeding (7-14). Recently, introduction of transrectal ultrasound (TRUS)-guided transperineal (TP) biopsy led to evaluation of the TP route for biopsy purposes, which provides direct access to the peripheral apical region of the prostate, which is inaccessible via the TR route (15).
However, the effect of interruption of antithrombotic agent administration before TP biopsy remains controversial. The primary goal of this study was to evaluate the safety of continued administration of antithrombotic agents during TP biopsy.

\section{PATIENTS AND METHODS}

Patients

After obtaining informed consent, 832 consecutive patients underwent TRUS-guided systematic TP biopsy to evaluate elevated PSA levels ( $>4 \mathrm{ng} / \mathrm{mL}$ ) and/or abnormal DRE findings at two Japanese institutions between July 2008 and August 2012. Patients with biochemical recurrence after prostatectomy $(\mathrm{n}=11)$ or radiation therapy $(n=2)$, those with fewer than 5 cores obtained by biopsy because of high suspicion of prostate cancer $(n=5)$, and those with simultaneous transurethral resection of bladder tumor and prostate biopsy $(n=3)$ were excluded. Thus, 811 patients were eligible for inclusion in the present retrospective study. This study was approved by the institutional review boards of both institutions.

Table-1 lists the clinical variables examined in the three groups of patients, including age,

Table 1 - Clinical characteristics of all 811 patients and 84 pairs of patients after propensity score matching (PSM).

\begin{tabular}{|c|c|c|c|c|c|c|c|}
\hline & \multicolumn{4}{|c|}{ All patients } & \multicolumn{3}{|c|}{ Matched patients } \\
\hline & $\begin{array}{c}\text { Group I } \\
(\mathrm{n}=672)\end{array}$ & $\begin{array}{l}\text { Group II } \\
(n=103)\end{array}$ & $\begin{array}{l}\text { Group III } \\
(n=36)\end{array}$ & $P$-value & $\begin{array}{l}\text { Group I } \\
(n=84)\end{array}$ & $\begin{array}{l}\text { Group II } \\
(n=84)\end{array}$ & $P$-value \\
\hline \multicolumn{8}{|l|}{ Median (IQR) } \\
\hline Age (years) & $69(63-74)$ & $72(67-77)$ & $70(64-74)$ & $<0.0001$ & $71(64-76)$ & $72(66-77)$ & 0.23 \\
\hline PSA (ng/mL) & $7.5(5.2-11.9)$ & $8.4(5.6-15.7)$ & $9.7(6.1-15.0)$ & 0.043 & $7.9(5.0-15.9)$ & $7.6(5.2-13.1)$ & 0.82 \\
\hline Total Prostate volume (mL) & $33(25-49)$ & $35(26-47)$ & $32.0(22-51)$ & 0.68 & $33(26-51)$ & $35(25-46)$ & 0.69 \\
\hline INR & $1.00(0.97-1.04)$ & $1.03(0.98-1.09)$ & $1.02(0.97-1.12)$ & $<0.0001$ & $1.01(0.96-1.05)$ & $1.02(0.98-1.06)$ & 0.13 \\
\hline Biopsy cores & $14(14-14)$ & $14(14-14)$ & $14(14-14)$ & 0.74 & $14(14-14)$ & $14(14-14)$ & 0.96 \\
\hline IPSS & $9(4-16)$ & $12(8-16)$ & $9(5-15)$ & 0.10 & $11(6-18)$ & $12(7-17)$ & 0.73 \\
\hline $\mathrm{BMI}\left(\mathrm{kg} / \mathrm{m}^{2}\right)$ & $23.0(21.2-24.7)$ & $23.5(21.6-25.1)$ & $22.3(21.7-24.2)$ & 0.11 & $23.4(21.7-25.1)$ & $23.6(21.7-25.3)$ & 0.41 \\
\hline \multicolumn{8}{|l|}{ N (\%) } \\
\hline Local anesthesia & $623(92.7)$ & $100(97.1)$ & $32(88.9)$ & 0.16 & $81(96.4)$ & $81(96.4)$ & 1.0 \\
\hline Abnormal DRE & $123(20.6)$ & $29(34.9)$ & $10(29.4)$ & 0.0086 & $25(29.8)$ & $28(33.3)$ & 0.70 \\
\hline
\end{tabular}


PSA level, total prostate volume, international normalized ratio (INR), biopsy cores, International Prostate Symptom Score (IPSS), body mass index (BMI), type of anesthesia, and DRE findings. The control group (group I) of 672 patients had never taken antithrombotic agents. Group II included patients who underwent TP biopsy while continuing antithrombotic therapy $(n=103)$, including 78 patients on an antiplatelet agent (56 on aspirin, 1 on clopidogrel, and 21 on another agent), 15 on an anticoagulant (warfarin), seven on dual antiplatelet agents, and three on both the antiplatelet and anticoagulant (Table-2). Because the number of patients taking anticoagulant was too small to analyze, we combined those patients with the antiplatelet group, although these drugs have different mechanisms of action and produce a different biologic response. In group III $(n=36)$, antithrombotic therapy was interrupted in accordance with a decision by the attending physician or at the patient's request. These agents were discontinued 7-10 days before TP biopsy and were resumed 1-2 days after completion of the procedure.

\section{Biopsy protocol}

A cleaning enema was administered before TP biopsy. For antimicrobial prophylaxis, levofloxa-

Table 2 - The detail of continuing anti-thrombotic agents.

\begin{tabular}{lc}
\hline & No. of patients \\
\hline Single agent & 56 \\
Aspirin & 4 \\
Cilostazol & 1 \\
Clopidogrel & 5 \\
Ticlopidine & 15 \\
Warfarin & 12 \\
Others & \\
Dual agents & 3 \\
Aspirin and cilostazol & 3 \\
Aspirin and clopidogrel & 1 \\
Aspirin and ticlopidine & 2 \\
Aspirin and warfarin & 1 \\
Warfarin and cilostazol & \\
\hline
\end{tabular}

cin (500mg) or tosufloxacin (300mg) was orally administered before the procedure. In general, the biopsy protocol included a standard systematic 14-core scheme, as described by Kawakami et al. (Figure-1) (15). When abnormal findings were observed during pre-biopsy MRI or TRUS, additional targeted biopsies were performed. With the patient in the lithotomy position, the prostate gland was imaged using an ultrasound machine (ALOKA SSD2000, Aloka, Tokyo, Japan) equipped with a biplanar 5/7.5-MHz TR probe (ALOKA UST- 672), and TRUS-guided TP biopsy was performed using a disposable 18-gauge needle (C.R. Bard, Inc., Covington, GA, USA). TP biopsy was performed using local anesthesia consisting of 1\% lidocaine $(\mathrm{n}=756)$ as described previously (16), spinal anesthesia $(n=52)$, or general anesthesia $(n=3)$. Biopsies were performed by experienced urologists (6 surgeons, mean experience of 17 years, range 6-32 years). After the procedure, the patients were hospitalized for 1 day to monitor and for treatment of any complications (i.e. acute urinary retention, hematuria or fever).

\section{Morbidity assessment}

All patients were evaluated around 2 weeks after the TP biopsy at the first outpatient visit, during which they were asked about the incidence of bleeding-associated events and other complications. Bleeding-associated events included gross hematuria, perineal hematoma, and rectal bleeding. These events noticed at least once were included. Gross hematuria was defined as passing blood or red urine. Perineal hematoma was defined as a non-pulsatile mass $>1.5 \mathrm{~cm}$ in diameter diagnosed by visual examination and palpation of the perineum. Rectal bleeding was defined as the passage of bright blood on or in the stool due to needle penetration through the rectum. The incidence of hematospermia was not evaluated due to lack of data.

Overall complications were graded as follows: grade 1 (need for hospital admission without medication), grade 2 (need for any type of intervention including medication, catheterization or blood transfusion), or grade 3 (life-threatening complications resulting in intensive care unit management or death). 
Figure 2 - 14-core scheme, as described by Kawakami et al.

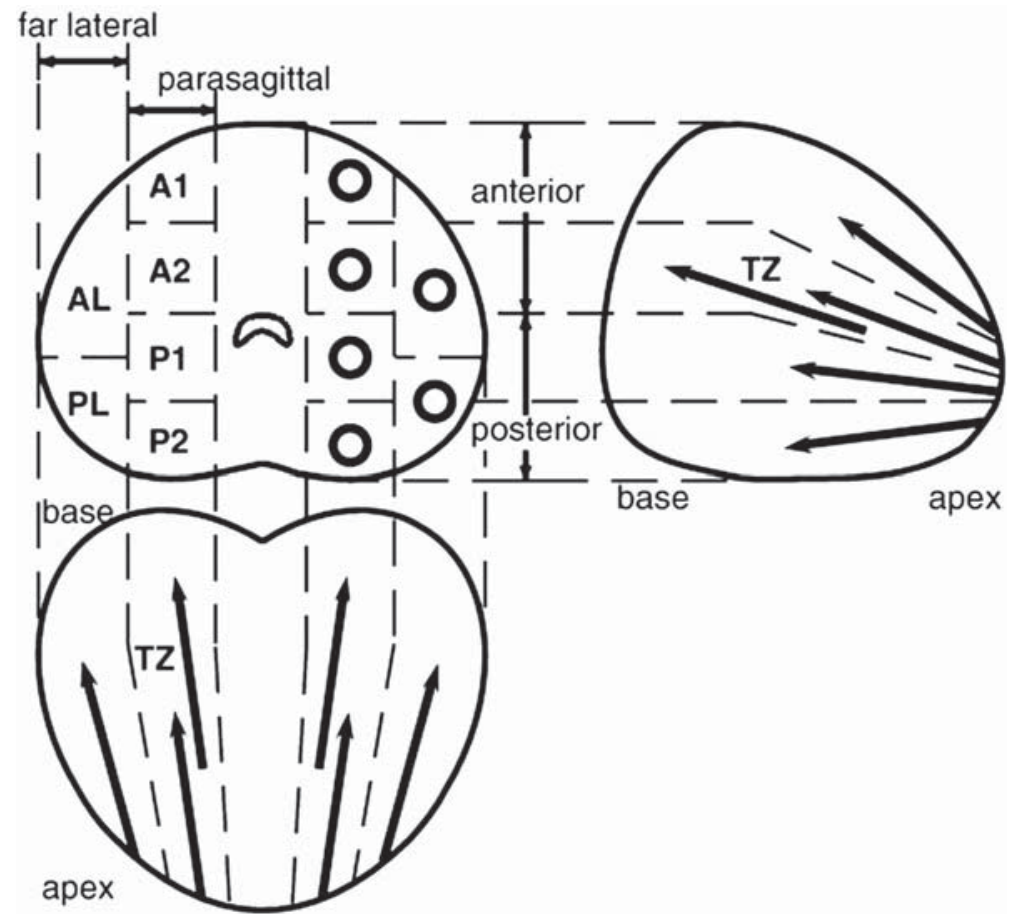

\section{Statistical analysis}

Comparisons between the three groups were performed using the Mann-Whitney U test or Kruskal-Wallis test for continuous variables and the chi-square test or Fisher's exact test for categorical variables. Univariate logistic regression analysis was used to identify the individual clinical factors predictive of hemorrhagic complications.

Given the non-randomized nature of the study, we used propensity score matching (PSM) analysis to compare hemorrhagic events and complications between groups I and II for determining the risk of continuing antithrombotic agents during TP biopsy. Group III was excluded from the analysis as it was difficult to precisely assess the relationship between resumption of antithrombotic agents and hemorrhagic complications. PSM was applied to correct for potential selection bias and to adjust for differences in baseline clinical characteristics. To derive the propensity score in this study, patient characteristics were entered into a logistic regression model. We used the greedy nearest-neighbor matching method within a caliper of 0.2 of the standard deviation $(17,18)$. Matching factors included age, BMI, DRE findings, PSA level, total prostate volume, INR, IPSS, and type of anesthesia.

Statistical analyses were performed using JMP 9.0.2 (SAS Institute Inc., Cary, NC, USA) and the $\mathrm{R}$ 2.15.2. statistical system ( $\mathrm{R}$ Foundation for Statistical Computing, Vienna, Austria). All P-values $<0.05$ was considered statistically significant.

\section{RESULTS}

Histological examination revealed the presence of prostate cancer in 383 patients (47.2\%). Of 383 patients, the biopsy Gleason score was 5-6 in $61(16.4 \%)$ patients, 7 in $181(48.5 \%)$ patients, and 8-10 in 131 (35.1\%) patients. Table-1 lists the characteristics of controls, patients continuing antithrombotic agents, and the interrupted anti- 
thrombotic agents groups before and after PSM. The matching analysis resulted in 84 well-matched pairs of group I and II patients. There were no significant differences in the clinical variables among the 811 patients in the three groups, except for age, PSA level, INR, and DRE findings. In group II, higher age, longer INR, and more frequent abnormal DRE findings were observed.

Table-3 showed the incidence of complications among the three groups. Of the 811 patients included in this study, complications occurred in 37 patients (4.6\%). Grade 1, 2, and 3 complications occurred in $12(1.4 \%), 24(2.9 \%)$, and one $(0.1 \%)$ patients, respectively. The most frequent biopsy-related complication was urinary retention (1.2\%). Although hemorrhagic compli- cations occurred in $15(1.8 \%)$ patients, many were grade 1 (73.3\%) and no grade 3 complications occurred. One patient in group III whose aspirin treatment was interrupted 7 days before TP biopsy, was transferred to the emergency department 10 days after the procedure. The patient was diagnosed with cerebral infarction requiring intensive care unit treatment.

Table- 4 showed the incidence of gross hematuria, perineal hematoma, and rectal bleeding of 168 matched patients compared with group I and group II. PSM analysis revealed that there were no significant differences in the incidence of all events between groups I and II. The incidence of hemorrhagic complications was similar in group I (2/84 patients) and group II (3/84 patients) after PSM.

Table 3 - Incidence of complications among the three groups.

\begin{tabular}{|c|c|c|c|c|c|c|c|c|c|c|}
\hline \multirow{3}{*}{ Complications } & \multicolumn{3}{|c|}{$\begin{array}{c}\text { Group I } \\
(\mathrm{n}=672)\end{array}$} & \multicolumn{3}{|c|}{$\begin{array}{l}\text { Group II } \\
(n=103)\end{array}$} & \multicolumn{3}{|c|}{$\begin{array}{l}\text { Group III } \\
(\mathrm{n}=36)\end{array}$} & \multirow[t]{3}{*}{ total } \\
\hline & \multicolumn{9}{|c|}{ Complication grade } & \\
\hline & G1 & G2 & G3 & G1 & G2 & G3 & G1 & G2 & G3 & \\
\hline Urinary retention & & 9 & & & 1 & & & & & 10 \\
\hline Acute prostatitis & & 5 & & & 1 & & & & & 6 \\
\hline Perineal pain & & 3 & & & & & & & & 3 \\
\hline Ischemic colitis & & 1 & & & & & & & & 1 \\
\hline Vasovagal reflex & 1 & & & & & & & & & 1 \\
\hline Cerebral infarction & & & & & & & & & 1 & 1 \\
\hline \multicolumn{11}{|l|}{ Hemorrhagic complication } \\
\hline Hematuria & 4 & & & 2 & & & 1 & & & 7 \\
\hline Colt retention & & 2 & & & 1 & & & & & 3 \\
\hline Perineal hematoma & 3 & 1 & & 1 & & & & & & 5 \\
\hline
\end{tabular}

Table 4 - Incidence of bleeding associated events after PSM.

\begin{tabular}{lccc}
\hline & $\begin{array}{c}\text { Group I } \\
(\mathrm{n}=84)\end{array}$ & $\begin{array}{c}\text { Group II } \\
(\mathrm{n}=84)\end{array}$ & P-value \\
\hline Gross hematuria & $39(46.4)$ & $38(45.2)$ & 0.88 \\
Perineal hematoma & $0(0)$ & $2(6.1)$ & 0.15 \\
Rectal bleeding & $0(0)$ & $0(0)$ & 1.0 \\
\hline
\end{tabular}


Lastly, we evaluated the predictive factors for hemorrhagic complications in the 811 patients (Table-5). Univariate analysis revealed that hemorrhagic complications were significantly associated with a $\mathrm{BMI}<21 \mathrm{~kg} / \mathrm{m}^{2}(\mathrm{P}=0.0058)$.

\section{COMMENTS}

We conducted a retrospective study including 811 patients in whom TRUS-guided TP biopsy was performed with or without continuation of antithrombotic agents during the procedure. Our study demonstrated that antithrombotic agents did not increase the frequency of bleeding-associated events or the risk of hemorrhagic complications during TP biopsy.

An extended life span is accompanied by an increase in the incidence of prostate cancer among elderly patients with a history of cardiovascular disease. Antithrombotic agents are routinely administered to these patients to prevent thromboembolism. Until recently, antithrombotic agents were routinely interrupted before diagnostic prostate biopsy to avoid hemorrhagic complications; however, thromboembolism triggered by discontinuation of antithrombotic agents may have life-threatening consequences. Of 36 patients in whom treatment with antithrombotic agents was interrupted in this study, cerebral infarction occurred in one, who required treatment in the intensive care unit. Life-threatening risk of drug interruption-associated thromboembolism may prompt clinicians to not discontinue antithrombotic agents prior to prostate biopsy; however, the risk of drug continuation in relation to biopsy-related hemorrhagic complication remains to be determined.

The TP route has been less often employed than TR route because it appears more invasive than TR biopsy $(19,20)$. Recently, the introduction of TRUS-guided extended TP biopsy increased the use of the TP route because of its much lower risk of infection, rectal bleeding and potential for improved sampling from the anterior portion of the prostate, which may not be accessible via the TR route $(21,22)$. Although perineal hematoma is a TP biopsy-specific complication, severe cases requiring blood transfusion or endoscopic intervention have not been reported. The absence of a free cavity between the prostate and perineum may be associated with a low hemorrhagic risk after TP biopsy.

Table 5 - Univariate logistic regression analysis for predicting the risk of hemorrhagic complications.

\begin{tabular}{lcc}
\hline Variable & Odds ratio $(95 \% \mathrm{Cl})$ & P-value \\
\hline Age, years $(\geq 70$ vs $<70)$ & $1.48(0.51-4.52)$ & 0.47 \\
PSA $(\geq 10 \mathrm{ng} / \mathrm{mL}$ vs. $<10 \mathrm{ng} / \mathrm{mL})$ & $0.48(0.11-1.52)$ & 0.23 \\
Total prostate volume $(\geq 30 \mathrm{~mL}$ vs. $<30 \mathrm{~mL})$ & $1.74(0.59-6.31)$ & 0.35 \\
INR $(\geq 1.04$ vs. $<1.04)$ & $2.72(0.97-7.85)$ & 0.058 \\
Biopsy cores $(\geq 15$ vs. $<15)$ & $\mathrm{N} / \mathrm{A} \dagger$ & 0.37 \\
IPSS $(\geq 9$ vs. $<9)$ & $0.23(0.01-1.44)$ & 0.18 \\
BMI $\left(<21 \mathrm{~kg} / \mathrm{m}^{2}\right.$ vs. $\left.\geq 21 \mathrm{~kg} / \mathrm{m}^{2}\right)$ & $4.52(1.55-13.89)$ & 0.0058 \\
Local anesthesia (local vs. others) & $1.04(0.20-18.99)$ & 0.97 \\
Abnormal DRE & $0.73(0.24-2.68)$ & 0.60 \\
Continuing anti-thrombotic agent (yes vs no) & $2.56(0.70-7.65)$ & 0.11 \\
\hline
\end{tabular}

†N/A, not applicable. 
In the present study, hemorrhagic complications occurred in 1.8\% of patients and most were self-remitting. According to the PSM analysis, no statistically significant difference was found in the incidence of gross hematuria, perineal hematoma, and rectal bleeding between controls (group I) and patients who continued antithrombotic agents (group II). Univariate analysis revealed that hemorrhagic complications were not associated with antithrombotic agents. These data indicate that hemorrhagic complications are rare after TP biopsy. Continued administration of antithrombotic agents does not increase the risk of hemorrhagic events or complications.

Our findings are consistent, in part, with the findings of Kariotis et al. (11), which indicated that younger patients with lower BMI receiving aspirin were at higher risk of developing hematuria after TR biopsy. It was assumed that patients with lower BMI were more sensitive to aspirin than obese patients (23). In this study, lower BMI $\left(<21 \mathrm{~kg} / \mathrm{m}^{2}\right)$ was associated with hemorrhagic complications in all patients. This result suggests that patients with low BMI require careful attention and monitoring after TP biopsy.

This study has several limitations. First, this is a retrospective study, and although we utilized PSM analysis, this does not replace randomization. Second, the sample size after PSM reduced. Among 103 patients with continued antithrombotic agents, only 84 were eligible for evaluation of the incidence of bleeding-associated events and hemorrhagic complications. A major weakness of PSM is the reduction in the number of participants to avoid poor matching pairs. Third, patient complications were not followed up for $>2$ weeks after the biopsy; thus, the possibility of late-onset complications cannot be ruled out.

\section{CONCLUSIONS}

Continued administration of antithrombotic agents does not increase the risk of hemorrhagic complications. It is not necessary to discontinue antithrombotic agents during TP biopsy. Further multicenter prospective randomized controlled trials are needed to validate the safety of continuing antithrombotic treatment.

\section{ABBREVIATIONS}

$\mathrm{BMI}=$ body mass index

DRE $=$ rectal examination

INR = international normalized ratio

IPSS = International Prostate Symptom Score

MRI = magnetic resonance imaging

PSA $=$ serum prostate-specific antigen

PSM = propensity score matching

$\mathrm{TP}=$ transperineal

$\mathrm{TR}=$ transrectal

TRUS $=$ transperineal ultrasound

\section{CONFLICT OF INTEREST}

None declared.

\section{REFERENCES}

1. Jemal A, Bray F, Center MM, Ferlay J, Ward E, Forman D. Global cancer statistics. CA Cancer J Clin. 2011;61:69-90. Erratum in: CA Cancer J Clin. 2011;61:134.

2. Connor SE, Wingate JP. Management of patients treated with aspirin or warfarin and evaluation of haemostasis prior to prostatic biopsy: a survey of current practice amongst radiologists and urologists. Clin Radiol. 1999;54:598-603.

3. Zhu JP, Davidsen MB, Meyhoff HH. Aspirin, a silent risk factor in urology. Scand J Urol Nephrol. 1995;29:369-74.

4. Maulaz AB, Bezerra DC, Michel P, Bogousslavsky J. Effect of discontinuing aspirin therapy on the risk of brain ischemic stroke. Arch Neurol. 2005;62:1217-20.

5. Wahl MJ. Myths of dental surgery in patients receiving anticoagulant therapy. J Am Dent Assoc. 2000;131:77-81.

6. Metersky ML, Nathanson I. Introducing the future of ACCP Clinical Practice Guidelines. Chest. 2012;141:285-6.

7. Maan Z, Cutting CW, Patel U, Kerry S, Pietrzak P, Perry MJ, et al. Morbidity of transrectal ultrasonography-guided prostate biopsies in patients after the continued use of low-dose aspirin. BJU Int. 2003;91:798-800.

8. Ihezue CU, Smart J, Dewbury KC, Mehta R, Burgess L. Biopsy of the prostate guided by transrectal ultrasound: relation between warfarin use and incidence of bleeding complications. Clin Radiol. 2005;60:459-63; discussion 457-8.

9. Giannarini G, Mogorovich A, Valent F, Morelli G, De Maria $M$, Manassero $F$, et al. Continuing or discontinuing lowdose aspirin before transrectal prostate biopsy: results of a prospective randomized trial. Urology. 2007;70:501-5. 
10. Halliwell OT, Yadegafar G, Lane C, Dewbury KC. Transrectal ultrasound-guided biopsy of the prostate: aspirin increases the incidence of minor bleeding complications. Clin Radiol. 2008;63:557-61.

11. Kariotis I, Philippou P, Volanis D, Serafetinides E, Delakas D. Safety of ultrasound-guided transrectal extended prostate biopsy in patients receiving low-dose aspirin. Int Braz J Urol. 2010;36:308-16.

12. Raheem $O A$, Casey RG, Galvin DJ, Manecksha RP, Varadaraj $\mathrm{H}$, McDermott $\mathrm{T}$, et al. Discontinuation of anticoagulant or antiplatelet therapy for transrectal ultrasound-guided prostate biopsies: a single-center experience. Korean J Urol. 2012;53:234-9.

13. Carmignani L, Picozzi S, Bozzini G, Negri E, Ricci C, Gaeta $M$, et al. Transrectal ultrasound-guided prostate biopsies in patients taking aspirin for cardiovascular disease: A metaanalysis. Transfus Apher Sci. 2011;45:275-80.

14. Chowdhury R, Abbas A, Idriz S, Hoy A, Rutherford EE, Smart JM. Should warfarin or aspirin be stopped prior to prostate biopsy? An analysis of bleeding complications related to increasing sample number regimes. Clin Radiol. 2012;67:e64-70.

15. Kawakami S, Kihara K, Fujii Y, Masuda H, Kobayashi T, Kageyama $Y$. Transrectal ultrasound-guided transperineal 14-core systematic biopsy detects apico-anterior cancer foci of T1c prostate cancer. Int J Urol. 2004;11:613-8.

16. Kubo $\mathrm{Y}$, Kawakami S, Numao N, Takazawa R, Fujii $\mathrm{Y}$, Masuda $\mathrm{H}$, et al. Simple and effective local anesthesia for transperineal extended prostate biopsy: application to threedimensional 26-core biopsy. Int J Urol. 2009;16:420-3.

17. Rosenbaum PR, Rubin DB. Constructing a control group using multivariate matched sampling methods that incorporate the propensity score. The American Statistician 1985;39: 33-8.
18. D’Agostino RB Jr. Propensity score methods for bias reduction in the comparison of a treatment to a nonrandomized control group. Stat Med. 1998;17:2265-81.

19. Noguchi M, Matsuoka K, Koga H, Kanetake H, Nakagawa $\mathrm{M}$, Naito $\mathrm{S}$. A questionnaire survey of patient preparation and techniques for prostate biopsy among urologists in the Kyushu and Okinawa regions of Japan. Int J Clin Oncol. 2006;11:390-5.

20. Shandera KC, Thibault GP, Deshon GE Jr. Variability in patient preparation for prostate biopsy among American urologists. Urology. 1998;52:644-6.

21. Chun FK, Epstein JI, Ficarra V, Freedland SJ, Montironi R, Montorsi F, et al. Optimizing performance and interpretation of prostate biopsy: a critical analysis of the literature. Eur Urol. 2010;58:851-64.

22. Kawakami S, Yamamoto S, Numao N, Ishikawa Y, Kihara $\mathrm{K}$, Fukui I. Direct comparison between transrectal and transperineal extended prostate biopsy for the detection of cancer. Int J Urol. 2007;14:719-24.

23. Greenblatt DJ, Abernethy DR, Boxenbaum HG, Matlis R, Ochs HR, Harmatz JS, et al. Influence of age, gender, and obesity on salicylate kinetics following single doses of aspirin. Arthritis Rheum. 1986;29:971-80.
Correspondence address:

Toko Asano, MD

Omori Red Cross Hospital - Urology 4-30-11, Chuo, Ota-ku Tokyo, 143-8527, Japan E-mail: touko_bg7@hotmail.co.jp 